\title{
Clinicopathological differences of colorectal cancers according to tumor origin: Identification of possibly de novo lesions
}

\author{
PETROS C. PAPAGIORGIS ${ }^{1}$, ADAMANTIA E. ZIZI ${ }^{2}$, SOPHIA TSELENI ${ }^{3}$, \\ IOANNIS N. OIKONOMAKIS ${ }^{4}$ and NIKOLAOS I. NIKITEAS ${ }^{5}$
}

\author{
${ }^{1}$ Department of Surgery, Athens Medical Center, Marousi $15125 ;{ }^{2}$ Department of Pathology, Tzaneio General Hospital, \\ Piraeus 185 36; ${ }^{3}$ Department of Pathology, Medical School, University of Athens, Athens 115 27; ${ }^{4}$ Department of Surgery, \\ 401 Army General Hospital, Athens 115 25; ${ }^{5}$ Second Department of Propedeutic Surgery, \\ Medical School, University of Athens, Athens 115 27, Greece
}

Received June 15, 2012; Accepted September 14, 2012

DOI: $10.3892 /$ br.2012.17

\begin{abstract}
Colorectal cancer (CRC) is considered to develop through the conventional adenoma-carcinoma sequence. However, the existence of de novo carcinogenesis, without any intervening precursor lesions, has been suggested for certain morphologically different tumors lacking polypoid characteristics. The presence of such tumors, along with their correlation with cardinal clinicopathological parameters, such as stage, grade and site, was retrospectively investigated in a series of 119 surgically treated CRC cases. The absence of particular polypoid characteristics (adenomatous remnants or coexisting polyps in the tumor vicinity) in combination with an infiltrative (or ulceroinfiltrative) growth pattern, were the criteria defining the nonpolypoid origin. The recorded frequencies of remnants, coexisting polyps and infiltrative tumors were $7,5,9$ and $32 \%$, respectively. The incidence of cases meeting the above-mentioned criteria was $28.5 \%$. These nonpolypoid lesions exhibited a predilection for proximal anatomical site $(\mathrm{P}=0.04)$, probably associated with their infiltrative pattern. Most importantly, de novo lesions (unlike polypoid) were rarely found among cases with indolent tumor characteristics (stage I or grade I, $\mathrm{P}=0.008$ ), showing a considerably different overall pattern of distribution by stage and grade as compared to that of polypoid tumors $(\mathrm{P}=0.03)$. The fact that nonpolypoid CRCs appeared to be clinicopathologically different from their polypoid counterparts is supportive of possible de novo origin and suggestive of a likely worse clinical behavior. The impact of these findings should be investigated to determine potential applications in the diagnosis, treatment and surveillance of these lesions.
\end{abstract}

Correspondence to: Dr P.C. Papagiorgis, Department of Surgery, Athens Medical Center, Distomou 5-7, Marousi 151 25, Greece E-mail: ppapagiorg@teiath.gr

Key words: polypoid, nonpolypoid, colorectal cancer, de novo carcinogenesis

\section{Introduction}

Colorectal cancer (CRC), one of the most common malignancies in the Western world (1), is considered to develop from benign precursor lesions (i.e., adenomatous polyps) through the ademona-carcinoma sequence (2). This term refers to a progressive malignant transformation, the phenotypic expression of underlying stepwise genetic alterations, according to the model developed by Fearon and Vogelstein (3). Notwithstanding the existence of certain genetically different tumors following alternative tumorigenic pathways (with various precursor-serrated adenomas) (4), this model remains the dominant carcinogenic mechanism of CRC.

However, the hypothesis of another tumorigenic pathway, that of de novo carcinogenesis, suggesting the development of a tumor from normal (or intact) colonic mucosa, without the intervening step of an adenoma, has been a matter of discussion for decades (5-11). By definition, de novo lesions are characterized by the lack of any adenomatous remnant (i.e., the only indisputable evidence of the origin of adenoma) $(7,9)$. However, the application of this criterion is hampered by the obliteration of adenomatous elements during tumor growth. Therefore, their detection is confined to a minority of CRC $(\sim 15-20 \%)(6,7)$, mostly those diagnosed in early stages and thought to retain their initial structural characteristics $(7,9,10)$. This objective difficulty may explain the wide variation in the reported frequencies of de novo tumors in the literature (range, 1-80\%) (6-13). It also necessitates the implementation of additional criteria, potentially associated with these lesions, including small size $(<1$ or $2 \mathrm{~cm})$, the limited invasion of the bowel wall (T1 lesions) and, mostly, the nonpolypoid growth pattern (presenting in various forms: flat, depressed and infiltrative) (6-11). Despite the lack of absolute specificity for any of these criteria (9), their application may be helpful in the identification of de novo tumors $(6,7,10,13)$.

At the genetic level, de novo carcinogenesis has been associated with specific molecular characteristics, such as a reduced proportion of Ki-Ras mutation $(9,13-15)$ [a key genetic event of the adenoma-carcinoma sequence (3)]. Moreover, de novo lesions exhibited a predilection for proximal tumor location $(7,16)$, an association strongly suggesting genetic disparity, 
given the considerable predominance of the microsatellite instability (MSI) and $\mathrm{CpG}$ island methylator phenotype (CIMP) tumorigenic pathways among proximal tumors (4). Other genetic and epigenetic alterations correlated with de novo tumors have also been detected (15,17-19), although inconsistently $(9,11)$.

Clinically, de novo tumors may represent a more aggressive (fast-growing at an early stage) subtype of CRC $(14,15,20)$. Consequently, their identification may be important in the planning of treatment and follow-up $(10,12,14)$.

In this study, we investigated the presence of de novo tumors, particularly among cases referred for surgery [being the large majority of CRC (1)], using a combination of histomorphological criteria for their identification (see Materials and methods). We also examined their association with particular clinicopathological parameters affecting CRC prognosis (i.e., stage, grade and site).

\section{Materials and methods}

Study population. The sample initially examined included 147 CRC cases, surgically treated between 2000 and 2003 in the Second Surgical Department of Tzaneio Hospital in Piraeus (Piraeus, Greece). Subsequent to excluding cases with recurrences, hereditary cancer, synchronous tumors of double location and unclear pathology reports, 119 patients were finally deemed eligible for the conducted retrospective investigation. None of the 119 patients had undergone neoadjuvant therapy, not performed during the study period.

De novo determination. Tumor specimens were examined for de novo origin based on the following criteria: i) lack of adenomatous remnants; ii) absence of coexisting polyps in the surgical specimen over a $10 \mathrm{~cm}$ distance from the tumor (potentially implying 'field cancerization', i.e., carcinogenic molecular alterations of the intestinal epithelium close to the tumor site). Similar genetic alterations in cancers of other anatomic areas (head and neck) have been detected within this distance (15). The existence of polyps beyond this limit was considered coincidental (i.e., not correlated with the primary lesion) and therefore was not recorded; and iii) apparently nonpolypoid growth pattern, based on morphological and histological appearance, in particular infiltrative lesions (without overhanging edge and showing massive infiltration of tumor cells) (6), including even those with a relatively limited ulceration $(7,20)$ but always excluding protruding exophytic tumors (with overhanging edge) (6). The latter type was also identified on the basis of tumor thickness (at least 2-fold greater than that of the adjacent normal mucosa) (20).

Tumor size and extent of invasion were not included in the examining criteria since only three cases of lesions were $<2 \mathrm{~cm}$ or presented with T1 invasion, characteristics also thought to facilitate de novo investigation. Moreover, flat and depressed lesions were not identified in our sample. These tumors are usually found in early CRC (not invading beyond the submucosa), since in an advanced disease their characteristics are frequently altered, being virtually indistinguishable from those with polypoid origin (21).

Tumors fulfilling all the aforementioned criteria were considered nonpolypoid (possibly de novo). By contrast, cases
Table I. Clinicopathological characteristics.

\begin{tabular}{|c|c|c|}
\hline Characteristics & $\begin{array}{l}\text { No. of cases } \\
\qquad(n=119)\end{array}$ & $\%$ \\
\hline \multicolumn{3}{|l|}{ Age (years) } \\
\hline$<70$ & 56 & 47 \\
\hline$>70$ & 63 & 53 \\
\hline \multicolumn{3}{|l|}{ Gender } \\
\hline Male & 69 & 38 \\
\hline Female & 50 & 42 \\
\hline \multicolumn{3}{|l|}{ Site } \\
\hline Proximal & 36 & 30 \\
\hline Distal & 83 & 70 \\
\hline \multicolumn{3}{|l|}{ TNM stage } \\
\hline I & 12 & 10 \\
\hline II & 50 & 42 \\
\hline III & 44 & 37 \\
\hline IV & 13 & 11 \\
\hline \multicolumn{3}{|l|}{ Grade } \\
\hline Well & 7 & 6 \\
\hline Moderate & 103 & 86.5 \\
\hline Poor & 9 & 7.5 \\
\hline \multicolumn{3}{|l|}{ Combined stage-grade } \\
\hline Indolent (stage I, G1) & 16 & 13.5 \\
\hline Intermediate (stage II-III, G2) & 82 & 69 \\
\hline Unfavorable (stage IV, G3) & 21 & 17.5 \\
\hline \multicolumn{3}{|l|}{ Growth pattern } \\
\hline Exophytic & 23 & 20 \\
\hline Infiltrative & 38 & 32 \\
\hline Ulcerated or mixed & 58 & 48 \\
\hline Remnants & 9 & 7.5 \\
\hline Coexisting polyps & 11 & 9.0 \\
\hline Combined polypoid characteristics ${ }^{\mathrm{a}}$ & 8 & 7.0 \\
\hline
\end{tabular}

${ }^{a}$ Exophytic lesions with remnants or polyps.

exhibiting either remnants or an apparently exophytic growth pattern were designated as 'polypoid'. Lesions with coexisting polyps in their vicinity were also classified as 'polypoid', provided they were not infiltrative. Otherwise, they were classified as cases of 'unclear origin', a category comprising tumors without remnants but with a mixed or unclear gross configuration (mostly due to extended ulceration).

Therefore, our stratification is based on the tumor growth pattern [according to the Japanese classification for CRC (22), slightly modified by integrating infiltrative with ulceroinfiltrative and ulcerated with unclassified lesions] and in combination with the presence/absence of remnants and coexisting polyps.

Clinicopathological classification. Tumors were classified as stage I, II, III, IV and grade G1, G2, G3 (well, moderate and poor), following the TNM and World Health Organization (WHO) classifications, respectively. We also divided cases 
A

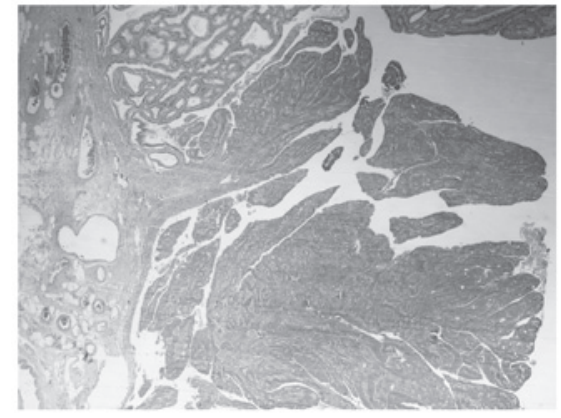

B

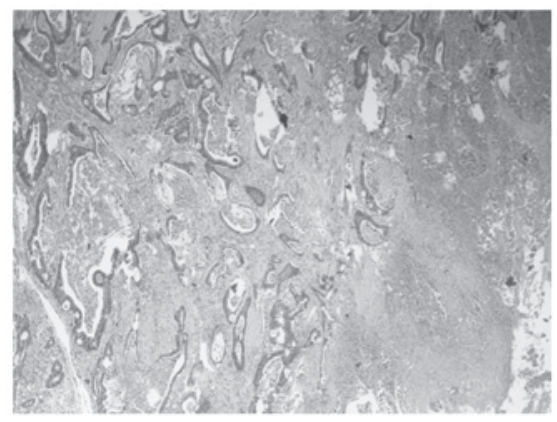

C

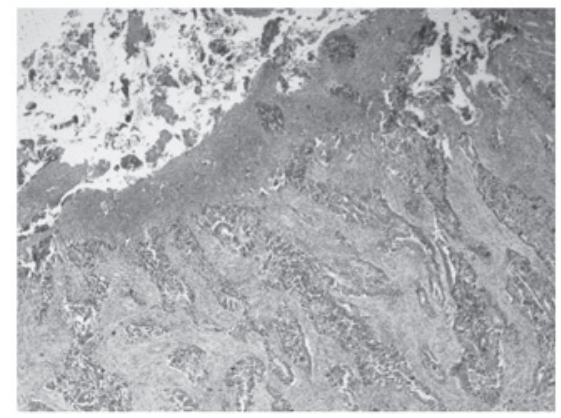

Figure 1. Histologic images (low power view) of (A) exophytic, (B) ulceroinfiltrative and (C) ulcerated adenocarcinomas. (A) Exophytic lesion accompanied by adenomatous remnant (magnification, $\mathrm{x} 20$ ) is shown. (B) Ulceroinfiltrative tumor (magnification, $\mathrm{x} 40$ ) is shown. (C) Ulcerated lesion (magnification, $\mathrm{x} 40$ ) is shown.

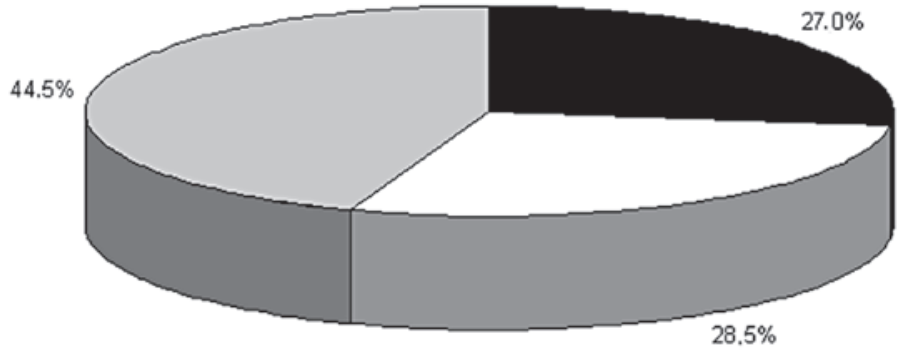

Polypoid aNonpolypoid aUnclear origin

Figure 2. Distribution of tumors according to their origin in our sample (119 cases). Polypoid (lesions with exophytic pattern and/or remnants or polyps), $27 \%$. Nonpolypoid (infiltrative lesions, without remnants or polyps), 28.5\%. Unclear origin (cases with extensive ulceration, mixed configuration or infiltrative lesions with coexisting polyps), $44.5 \%$.

into proximal (right-sided) and distal (left-sided), with regard to the splenic flexure $(7,16)$. Moreover, in order to examine the combined effect of stage and grade on de novo distribution, we stratified cases into three additional subsets: indolent (stage I or G1), unfavorable (stage IV or G3) and intermediate (stages II-III, G2). The absence of tumors with entirely conflicting characteristics (stage I/G3 or stage IV/G1) rendered any exclusion unnecessary.

Statistical analysis. The distribution of the particular criteria suggesting de novo origin among the various clinicopathological categories (stage, grade and site) was analyzed using the $\chi^{2}$ test (with Yates's correction when necessary) and Fisher's exact test, depending on the dataset. These tests were also used for the subsequent analysis of the distribution of tumors considered de novo (according to the aforementioned criteria) among the same clinicopathological categories. The tests were two-sided and $\mathrm{P} \leq 0.05$ was considered to indicate a statistically significant difference.

\section{Results}

Prevalence of the examining variables and classification according to tumor origin. Table I shows the clinicopathological characteristics of the sample as well as the detection rates of the parameters investigated in this study. Adenomatous remnants were detected in $9(7.5 \%)$, while coexisting polyps were observed in 11 cases $(9.2 \%)$. The recorded frequencies of the exophytic and infiltrative (or ulceroinfiltrative) growth patterns were 20 and $32 \%$, respectively. The remaining $48 \%$ of tumors exhibited a mixed or unclear growth pattern. Representative histologic images of lesions with remnants and of the various patterns are shown in Fig. 1.

Based on these results and according to the aforementioned criteria we classified $27 \%$ of the cases as 'apparently polypoid' (those with remnants, coexisting polyps and/or exophytic pattern), $28.5 \%$ as potentially de novo (infiltrative, always without remnants or coexisting polyps) and $44.5 \%$ as 'of unclear origin' (Fig. 2).

Associations of the examining variables with clinicopathological characteristics. As shown in Table II, remnants were more frequently detected among stage I tumors compared to the other stages in combination (II-IV, 33 vs. $5.5 \%, \mathrm{P}=0.003$ ) or individually (II, III and IV, P=0.01, 0.049 and 0.04 , respectively). The remnants also exhibited a trend towards well-differentiation ( 22 vs. $6 \%, \mathrm{P}=0.09$ ). These were observed in a markedly higher proportion in exophytic lesions compared to other growth patterns ( 22 vs. $4 \%, \mathrm{P}=0.015)$. Notably, this predilection remained significant in the particular comparison of exophytic tumors with infiltrative lesions $(\mathrm{P}=0.048)$, however, not with those exhibiting an unclear pattern $(\mathrm{P}=0.07)$. 
Table II. Distribution of remnants and co-existing polyps in various clinicopathological categories.

\begin{tabular}{|c|c|c|c|c|c|c|}
\hline \multirow[b]{2}{*}{ Category $(n)^{\mathrm{a}}$} & \multicolumn{3}{|c|}{ Remnants } & \multicolumn{3}{|c|}{ Polyps } \\
\hline & No. & Percentage & P-value & No. & Percentage & P-value \\
\hline \multicolumn{7}{|l|}{ Growth pattern } \\
\hline Exophytic (23) & 5 & 22 & $0.02^{\mathrm{b}}$ & 3 & 13 & $N S^{b}$ \\
\hline Infiltrative (38) & 1 & 2.6 & & 3 & 8 & \\
\hline Ulcerated (58) & 3 & 5 & & 5 & 8 & \\
\hline \multicolumn{7}{|l|}{ Stage } \\
\hline I (12) & 4 & 33 & $0.003^{\mathrm{b}}$ & 3 & 25 & $\mathrm{NS}\left(0.14^{\mathrm{b}}\right)$ \\
\hline II (50) & 2 & 4 & & 3 & 6 & \\
\hline III (44) & 3 & 7 & & 2 & 4.5 & \\
\hline IV (13) & - & 0 & & 3 & 23 & \\
\hline \multicolumn{7}{|l|}{ Grade } \\
\hline Well (G1) (7) & 2 & 29 & $\mathrm{NS}(0.09)^{\mathrm{b}}$ & - & 0 & $N S^{b}$ \\
\hline Moderate (G2) (103) & 6 & 6 & & 11 & 11 & \\
\hline Poor (G3) (9) & 1 & 11 & & - & 0 & \\
\hline \multicolumn{7}{|l|}{ Site } \\
\hline Proximal (36) & 3 & 8.5 & $N S^{b}$ & 2 & 5.5 & $N S^{b}$ \\
\hline Distal (83) & 6 & 7.2 & & 9 & 11 & \\
\hline Total (119) & 9 & 7.5 & & 11 & 9.2 & \\
\hline
\end{tabular}

${ }^{a}$ Total cases of each subset. Percentages in the next columns were calculated upon this number. ${ }^{b}$ First subset of each particular category (i.e. exophytic, stage I, G1, proximal) vs. other subsets, for instance, stage I vs. stages II-IV. NS, not significant.

A

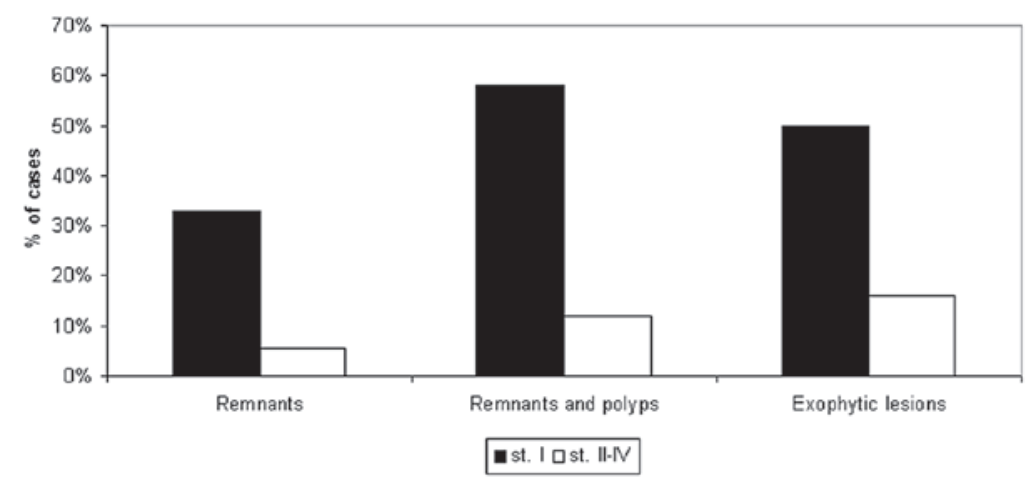

$\mathbf{B}$

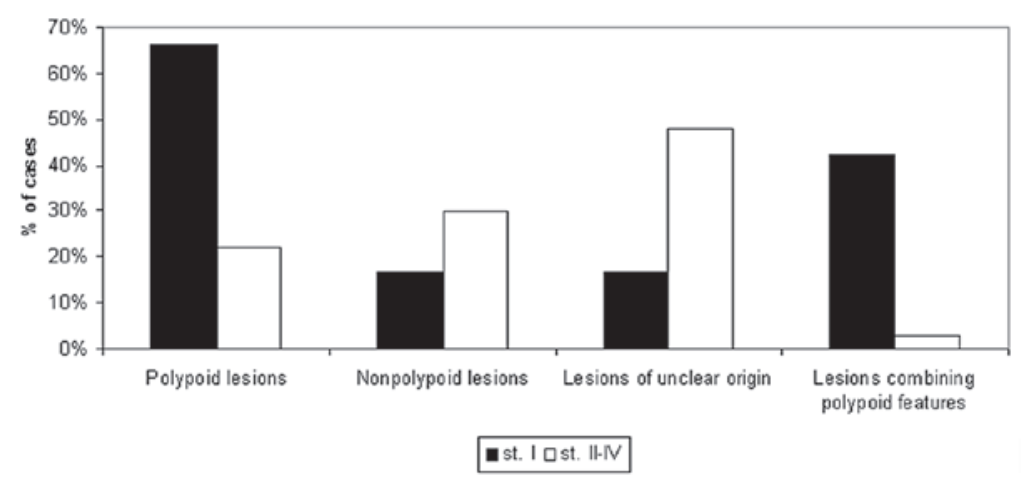

Figure 3. Comparison of various tumor characteristics (A) and categories of origin (B) between stage I and stages II-IV. (A) As indicated, the proportion of remnants, remnants and coexisting polyps or exophytic lesions was markedly higher in stage I compared to stages II-IV ( $\mathrm{P}=0.003,<0.001$ and 0.014 , respectively). (B) The prevalence of polypoid lesions was markedly higher in stage I compared to stages II-IV ( $\mathrm{P}=0.003)$. Conversely, lesions of an unclear origin were recorded in a markedly lower proportion in stage I compared to other stages $(\mathrm{P}=0.04)$. No statistically significant difference was observed in the incidence of de novo lesions between stage I and stages II-IV. Moreover, a considerably higher incidence of cases combining polypoid characteristics (exophytic pattern with remnants or polyps) was recorded in stage I ( 42 vs. $2.8 \%, \mathrm{P}<0,0001)$. 
Table III. Distribution of growth pattern by stage, grade and site.

\begin{tabular}{|c|c|c|c|c|c|c|c|c|c|}
\hline \multirow[b]{2}{*}{ Category $(\mathrm{n})^{\mathrm{a}}$} & \multicolumn{3}{|c|}{ Exophytic } & \multicolumn{3}{|c|}{$\begin{array}{l}\text { Growth pattern } \\
\text { Infiltrative/ulceroinfiltrative }\end{array}$} & \multicolumn{3}{|c|}{ Ulcerated and/or mixed } \\
\hline & No. & Percentage & P-value & No. & Percentage & P-value & No. & Percentage & P-value \\
\hline \multicolumn{10}{|l|}{ Stage } \\
\hline I (12) & 6 & 50.0 & $0.014^{\mathrm{b}}$ & 3 & 25.0 & $\mathrm{NS}^{\mathrm{b}}$ & 3 & 25.0 & $\mathrm{NS}^{\mathrm{b}}$ \\
\hline II (50) & 9 & 18.0 & & 14 & 28.0 & & 27 & 54.0 & \\
\hline III (44) & 6 & 13.5 & & 16 & 36.5 & & 22 & 50.0 & \\
\hline IV (13) & 2 & 15.5 & & 5 & 38.5 & & 6 & 46.0 & \\
\hline \multicolumn{10}{|l|}{ Grade } \\
\hline Well (7) & 3 & 43.0 & $0.1^{\mathrm{b}}$ & 2 & 28.5 & & 2 & 28.5 & $\mathrm{NS}^{\mathrm{b}}$ \\
\hline Moderate (103) & 20 & 19.5 & & 32 & 31.5 & & 51 & 50.0 & \\
\hline Poor (9) & - & 0.0 & $0.13^{\mathrm{c}}$ & 4 & 44.5 & $\mathrm{NS}^{\mathrm{c}}$ & 5 & 55.5 & $\mathrm{NS}^{\mathrm{c}}$ \\
\hline \multicolumn{10}{|l|}{ Site } \\
\hline Proximal (36) & 5 & 14.0 & $\mathrm{NS}^{\mathrm{b}}$ & 16 & 44.5 & $0.052^{\mathrm{b}}$ & 15 & 41.5 & $\mathrm{NS} \mathrm{S}^{\mathrm{b}}$ \\
\hline Distal (83) & 18 & 21.5 & & 22 & 26.5 & & 43 & 52.0 & \\
\hline Total (119) & 23 & 20.0 & & 38 & 32.0 & & 58 & 48.0 & \\
\hline
\end{tabular}

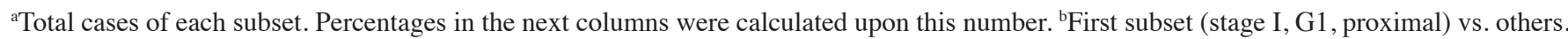
${ }^{\mathrm{c}}$ Poor grade vs. others. NS, not significant. $\mathrm{P}<0.15$, although not significant it was included in the table to indicate corresponding trends.

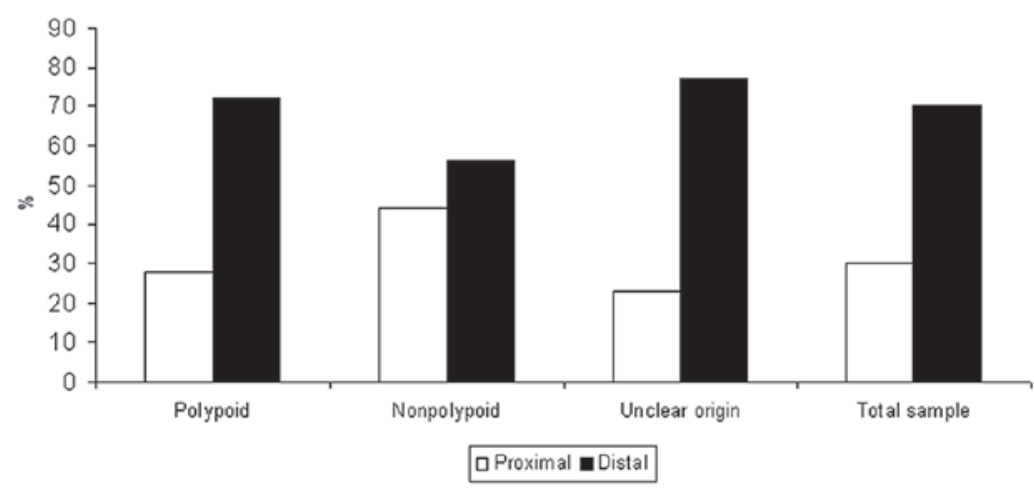

Figure 4. Segmental distribution according to tumor origin is shown. The proportion of proximal tumors was markedly higher among nonpolypoid tumors compared to that observed among polypoid and unclarified lesions $(\mathrm{P}=0.04)$, showing a relatively similar pattern of segmental distribution. Notably, while polypoid tumors were the subset better approaching the anatomical distribution of the total sample, nonpolypoid lesions were widely diverging from that distribution.

The presence of coexisting polyps was also more commonly recorded in stage I tumors. However, this trend was not statistically significant. In addition, no statistically significant difference was observed in the incidence of polyps in the other clinicopathological categories.

Moreover, remnants and polyps (considered together) displayed clear predilection, mostly for stage I disease (58 vs. $12 \%, \mathrm{P}<0.001$ ) and, to a lesser extent, for exophytic pattern (35 vs. $12,5 \%, \mathrm{P}=0.025)$. Also, while half of stage I tumors were exophytic, the incidence of this growth pattern among stages II-IV was only $16 \%(\mathrm{P}=0.014)$. Overall, polypoid characteristics (remnants, polyps and exophytic lesions) were detected in a markedly higher proportion in stage I compared to stages II-IV (66 vs. $22 \%, \mathrm{P}=0.003$ ). In addition, tumors combining these characteristics (exophytic lesions with remnants or polyps) were predominantly found in stage I (42 vs. $2.8 \%, \mathrm{P}<0.0001$ ) (Fig. 3).

The distribution of growth pattern by stage, grade and site is demonstrated in detail in Table III. Apart from the abovementioned findings regarding stage, exophytic lesions exhibited a trend for well-differentiation and a total lack of poor grade. However, the two findings were not statistically significant. Infiltrative lesions exhibited a tendency for proximal location $(\mathrm{P}=0.052)$ becoming significant for tumors finally classified as de novo $(\mathrm{P}=0.04)$. As shown in Fig. 4 , the pattern of segmental distribution in nonpolypoid cases was prominently different compared to polypoid and cases of unclear origin.

Clinicopathological distribution of de novo tumors. Table IV shows the distribution of lesions classified as de novo by stage, 


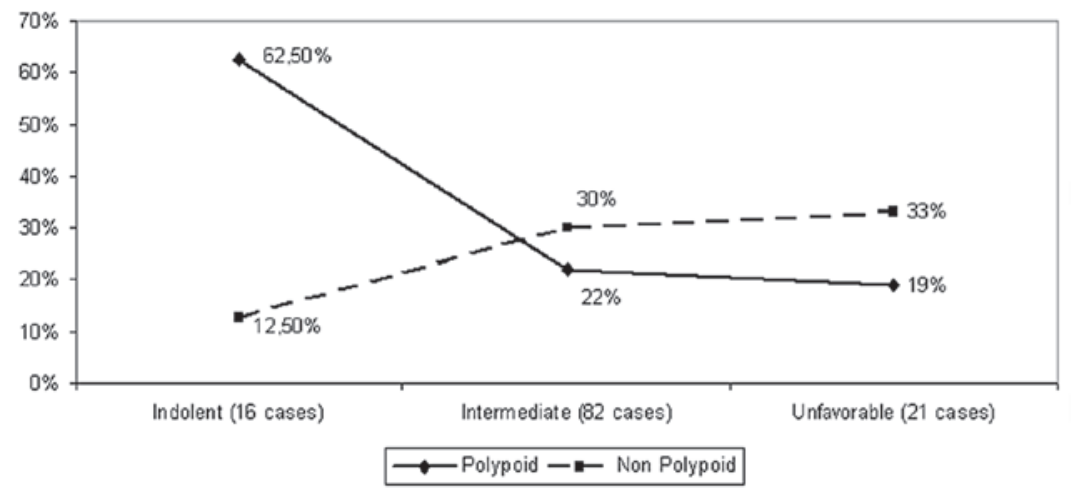

Figure 5. Disparate distribution of polypoid and non-polypoid lesions among combined stage-grade categories is shown. The frequency of polypoid tumors was reduced from $62.5 \%$ in the indolent subset to a mere $19 \%$ in the unfavorable category. Conversely, the proportion of non-polypoid lesions increased from 12.5 to $33 \%$ in the same categories $(\mathrm{P}=0.03$ for the comparison of overall distributions and $\mathrm{P}=0.008$ for the particular comparison of the observed proportions in the indolent subset).

grade and site, exhibiting the aforementioned predilection for proximal location as well as a higher (albeit not significantly) detection rate in cases with poor grade or unfavorable characteristics (stage IV or G3). However, the observed total pattern of distribution of these lesions varying between $12.5 \%$ (indolent cases) and 33\% (unfavorable cases) clearly differed from that observed for polypoid tumors, ranging from 62.5 to $19 \%$ in the same categories $(\mathrm{P}=0.03$, Fig. 5). This disparity was more evident in the indolent subset $(\mathrm{P}=0.008)$.

\section{Discussion}

The aim of this study was to identify de novo tumors on the basis of certain histomorphological criteria (lack of remnants or coexisting polyps, nonpolypoid configuration). We also examined their correlation with particular clinicopathological characteristics (stage, grade and site). The study was conducted in a sample mainly comprising 'advanced' CRC (i.e., tumors invading beyond the submucosa), consistent with certain previous studies $(7,12,13,20,23)$, although in contrast to most Japanese $(5,6,10,18,19)$ and certain Western studies $(8,11)$ focusing on early (T1) lesions.

Consistent with the findings reported by Chen et al, the ascertained incidence of potentially de novo tumors in our series was $28.5 \%$ (12). However, the incidence of such lesions is widely varying (even within a given study) according to the criteria occasionally used for their detection. In the large-scale study by Bedenne et al (7) de novo tumors (defined as those lacking remnants) were found in $40 \%$ of the cases, ranging from $17 \%$ in small $\mathrm{T} 1$ lesions to almost $100 \%$ in tumors with an endophytic (nonpolypoid) growth pattern. Largely diverging frequencies were also reported by Shimoda et al (6) (25\% in early and $80 \%$ in advanced lesions). Moreover, in the study by Goto et al (10), the proportion of de novo lesions was $23 \%$ in early (T1) CRC, increasing to $32 \%$ when small advanced lesions were additionally included in the analyzed sample. These discrepancies might be attributed in part to the difficulty in the colonoscopic detection of early de novo lesions and their potentially rapid growth, leading to their underestimation in early CRC and, concurrently to a diagnostic delay $(14,21)$ resulting in their overrepresentation in advanced CRC.
The presence of infiltrative growth pattern seems to be suggestive of de novo origin, as indicated by the rarity in the appearance of remnants among infiltrative lesions $(2.5 \%)$, consistent with previous results $(6,7)$. However, a number of those may actually arise from adenomas, with their gross configuration being altered into nonpolypoid during their evolution $(10,23)$, although this has been disputed $(6,13)$. Nevertheless, the effect of this bias (if any) may be counteracted by the potentially simultaneous presence of certain tumors with nonpolypoid route among cases classified as of unclear origin (due to their extended ulceration).

The low proportion (27\%) of lesions with apparently polypoid characteristics in our sample is probably not representative of the actual incidence of CRC of adenoma origin, although similar results have been occasionally reported $(6,24)$. Tumors of 'unclear origin', accounting for $44.5 \%$ of our cohort, likely arise from polyps (for the most part) (23), as possibly suggested by their predilection for distal tumor site (similar to polypoid and unlike de novo lesions). Supportive of this assumption was also the recorded high incidence of polypoid lesions in stage I (the earliest disease cases in our sample), $67 \%$ consistent with findings of a previous study in a similar subset (T2 lesions) (25). Moreover, this tendency towards earlier stage (considerably stronger for cases combining polypoid characteristics) is possibly indicative of more favorable behavior and/or easier colonoscopic detection of polypoid lesions and should be investigated in larger samples.

Consistent with results of previous studies $(7,16)$, we found lesions possibly developing de novo, in a significantly higher proportion among proximal tumors. This trend was particularly associated with the infiltrative growth pattern, more frequently observed proximally. Given the known clinicopathological and molecular differences of proximal CRC $(4,16,26,27)$, the recorded link between nonpolypoid lesions and proximal location supports the assumption of a different tumorigenic mechanism for de novo CRC. Moreover, this association suggests a higher malignant potential of de novo tumors, anticipated to the corresponding clinicopathological pattern reported for proximal tumors [higher stage and grade, worse outcome $(16,27,28)]$. The recorded rarity of de novo lesions in the indolent subset is also supportive of this state and consistent with previous findings $(20,29)$. It is also potentially 
Table IV. Distribution of de novo tumors in various clinicopathological categories.

De novo lesions

\begin{tabular}{lrcc}
\cline { 2 - 4 } Category (n) & No. & Percentage & P-value $^{\mathrm{b}}$ \\
\hline Stage & & & \\
I (12) & 2 & 17 & $\mathrm{NS}^{\mathrm{c}}$ \\
II (50) & 14 & 28 & \\
III (44) & 3 & 34 & \\
IV (13) & 23 & $\mathrm{NS}^{\mathrm{d}}$ \\
Grade & 28 & 29 & \\
Well (G1) (7) & 4 & 44 & \\
Moderate (G2) (103) & 28 & & \\
Poor (G3) (9) & & & \\
Site & 15 & 42 & \\
Proximal (36) & 19 & 23 & \\
Distal (83) & & & \\
Combined stage-grade & & 12.5 & \\
Indolent (16) & 2 & 30 & \\
Intermediate (82) & 25 & 33 & \\
Unfavorable (21) & 7 & 28.5 & \\
Total (119) & 34 & & \\
\hline
\end{tabular}

${ }^{\mathrm{a}}$ Total cases of each subset. Percentages in the next columns were calculated upon this number. ${ }^{\mathrm{b}}$ All comparisons were performed between the observed frequencies of non-polypoid lesions in the various categories. The distribution of polypoid and tumors of unclear origins

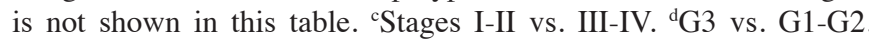
eProximal vs. distal. Indolent vs. intermediate-unfavorable. NS, not significant.

correlated with the proximal predilection of these tumors, considering the milder clinical manifestations and the lower efficacy of colonoscopy observed in proximal CRC, resulting in later stage presentation $(16,28)$.

From the clinical aspect, these results require more awareness and persistence in the colonoscopic detection of nonpolypoid lesions (particularly in the proximal segment), more intensive surveillance of the colonoscopically treated cases and surgical treatment for selected patients. These suggestions are also applicable for cancers alternatively evolving through a corresponding nonpolypoid adenoma, instead of direct de novo development (14). The clinical significance of nonpolypoid lesions, emphasized in recent studies $(21,30)$, is expected to be further elucidated by the ongoing prospective Japan Polyp Study (31), evaluating CRC surveillance strategies after initial colonoscopic removal of the detected neoplasia.

To the best of our knowledge, the current study is the first using the presence of coexisting polyps as a discriminative criterion between polypoid and nonpolypoid CRC. This application was based on the 'field cancerization theory', involving the dissemination of genetic changes characteristic of the primary tumor over a wide distance from the edges of the lesion $(15,32,33)$. Therefore, coexisting polyps, likely originating from the same changes, are indicative of a polypoid origin of the primary tumor. Additional investigation of the molecular alterations potentially occurring in the seemingly healthy colonic mucosa near the tumor is necessary to determine the exact distance required for the definite characterization of a given polyp as actually correlated with the primary lesion.

A limitation to our study was the relatively small sample size [although comparable to other relevant studies $(10,11,13,20,25)]$, potentially affecting results, particularly in subset analysis. Another limitation could be the almost complete lack of small and/or T1 lesions in our samples, potentially hampering de novo identification. However, such investigation in tumors invading beyond the submucosa (as we did), was also conducted by other scientists $(7,12,13,20,25)$, reporting frequencies of de novo lesions ranging from 11 to $40 \%$. Our findings $(28.5 \%)$ were within this range. Additionally, consistent with our findings, the incidence of nonpolypoid small $\mathrm{T} 1$ tumors has been found to be markedly low $(<5 \%)$ in previous studies $(9,11,16,34)$, suggesting a rather limited utility of the particular criteria for de novo identification (particularly among CRC referred for surgery).

In conclusion, our study was suggestive of a potential de novo origin for a considerable proportion of $\mathrm{CRC}$, concurrently indicating the predilection of these lesions for proximal tumor location and introducing an additional approach (that of coexisting polyps) potentially facilitating de novo identification. The clinical impact of our findings should be examined, especially with regard to possible associations between de novo lesions and important prognostic variables. The tendency for a worse behavior of nonpolypoid lesions, as suggested by their increasing incidence with disease progression and aggressiveness may necessitate adequate readjustments in the diagnosis, treatment and surveillance of these cases.

\section{Acknowledgements}

The authors would like to thank Dr F. Georgiadis for his help, Mrs. N. Vathi and Mr. V. Anthoulis for their valuable assistance in the preparation of the manuscript.

\section{References}

1. Jemal A, Siegel R, Ward E, Hao Y, Xu J, Murray T and Thun MJ: Cancer statistics. CA Cancer J Clin 58: 71-96, 2008.

2. Morson B: President's address. The polyp-cancer sequence in the large bowel. Proc R Soc Med 67: 451-457, 1974.

3. Fearon ER and Vogelstein B: A genetic model for colorectal tumorigenesis. Cell 6: 759-767, 1990.

4. Jass JR: Classification of colorectal cancer based on correlation of clinical, morphological and molecular features. Histopathology 50: 113-130, 2007.

5. Kuramoto $\mathrm{S}$ and Oohara T: Minute cancers arising de novo in the human large intestine. Cancer 61: 829-834, 1988.

6. Shimoda T, Ikegami M, Fujisaki J, Matsui T, Aizawa S and Ishikawa E: Early colorectal carcinoma with special reference to its development de novo. Cancer 64: 1138-1146, 1989.

7. Bedenne L, Faivre J, Boutron MC, Piard F, Cauvin MJ and Hillon P: Adenoma-carcinoma sequence or de novo carcinogenesis? A study of adenomatous remnants in population-based series of large bowel cancers. Cancer 69: 883-888, 1992.

8. Stolte M and Bethke B: Colorectal mini-de novo carcinoma: a reality in Germany too. Endoscopy 27: 286-290, 1995.

9. Mueller JD, Bethke B and Stolte M: Colorectal de novo carcinoma: a review of its diagnosis, histopathology, molecular biology, and clinical relevance. Virchows Arch 440: 453-460, 2002. 
10. Goto H, Oda Y, Murakami Y, et al: Proportion of de novo cancers among colorectal cancers in Japan. Gastroenterology 131: 40-46, 2006.

11. Hornic J, Farraye F and Odze R: Clinicopathologic and immunohistochemical study of small apparently 'de novo' colorectal adenocarcinomas. Am J Surgh Pathol 31: 207-215, 2007.

12. Chen CD, Yen MF, Wang WM, Wong JM and Chen TH: A case-cohort study for the disease natural history of adenoma-carcinoma and de novo carcinoma and surveillance of colon and rectum after polypectomy: implication for efficacy of colonoscopy. Br J Cancer 88: 1866-1873, 2003.

13. Kaneko K, Kurahashi T, Makino R, et al: Pathological features and genetic alterations in colorectal carcinomas with characteristics of nonpolypoid growth. Br J Cancer 91: 312-318, 2004.

14. Kashida $\mathrm{H}$ and Kudo S: Early colorectal cancer: concept, diagnosis, and management. Int J Clin Oncol 11: 1-8, 2006.

15. Tanaka T: Colorectal carcinogenesis: review of human and experimental animal studies. J Carcinog 8: 5, 2009.

16. Nawa T, Kato J, Kawamoto H, et al: Differences between right-and left-sided colon cancer in patient characteristics, cancer morphology and histology. J Gastroenderol Hepatol 23: 418-423, 2008.

17. Ogawa T, Yoshida T, Tsuruta T, Saigenji K and Okayasu I: Genetic instability on chromosome 17 in the epithelium of non-polypoid colorectal carcinomas compared to polypoid lesions. Cancer Sci 97: 1335-1342, 2006.

18. Nosho K, Yamamoto $\mathrm{H}$, Takahashi $\mathrm{T}$, et al: Genetic and epigenetic profiling in early colorectal tumors and prediction of invasive potential in pT1 (early invasive) colorectal cancers. Carcinogenesis 28: 1364-1370, 2007.

19. Yasugi A, Yashima K, Hara A, et al: Fhit, Mlh1, p53 and phenotypic expression in the early stage of colorectal neoplasms. Oncol Rep 19: 41-47, 2008.

20. Nasir A, Boulware D, Kaiser H, et al: Flat and polypoid adenocarcinomas of the colorectum: a comparative histomorphologic analysis of 47 cases. Hum Pathol 35: 604-611, 2004.

21. Soetikno R, Kaltenbach T, Rouse R, et al: Prevalence of nonpolypoid (flat and depressed) colorectal neoplasms in asymptomatic and symptomatic adults. JAMA 299: 1027-1035, 2008.

22. Yasutomi M, Baba S, Hojo K, et al (eds): Japanese classification of colorectal cancinoma. Kanehara \& Co, Ltd., Tokyo, 1997.

23. Matsui T, Yao T and Iwashita A: Natural history of early colorectal cancer. World J Surg 24: 1022-1028, 2000.
24. Nakamura K: De novo cancer and adenoma-carcinoma sequence of the colorectum-clinicopathological differences between de novo carcinoma and carcinoma with the sequence. Nihon Geka Gakkai Zaasshi 100: 766-775, 1999 (In Japanese).

25. Goi T, Kawasaki M, Hirono Y, Katayama K and Yamaguchi A: Clinicopathological analysis of invading muscularis propria (T2) cancers $\leq 20 \mathrm{~mm}$ in diameter. Int Surg 93: 1-5, 2008.

26. Sugai T, Habano W, Jiao YF, Tsukahara M, Takeda Y, Otsuka K and Nakamura S: Analysis of molecular alterations in left- and right-sided colorectal carcinomas reveals distinct pathways of carcinogenesis. J Mol Diagn 8: 193-201, 2006.

27. Papagiorgis PC,Zizi AE, Tseleni S, et al: Site impact on colorectal cancer biological behavior in terms of clinicopathological and molecular features. J BUON 16: 84-92, 2011.

28. Wong R: Proximal tumors are associated with greater mortality in colon cancer. J Gen Intern Med 25: 1157-1163, 2010.

29. Kurisu Y, Shimoda T, Ochiai A, Nakanishi Y, Hirata I and Katsu KI: Histologic and immunohistochemical analysis of early submucosal invasive carcinoma of the colon and rectum. Pathol Int 49: 608-616, 1999.

30. Matsuda T, Saito Y, Hotta K, Sano Y and Fujii T: Prevalence and clinicopathological features of nonpolypoid colorectal neoplasms: should we pay more attention to indentifying flat and depressed lesions? Dig Endoscopy 22: S57-S62, 2010.

31. Sano Y, Fujii T, Oda Y, et al: A multicenter randomized controlled trial designed to evaluate follow-up surveillance strategies for colorectal cancer: the Japan Polyp Study. Dig Endoscop 16: 376-378, 2004.

32. Dakubo GD, Jakupciak JP, Birch-Machin MA and Parr RL: Clinical implications and utility of field cancerization. Cancer Cell Int 7: 2, 2007

33. Aivado M, Gynes M, Golerov V, Schmidt WU, Röher HD and Goretzki PE: 'Field cancerization' - an additional phenomenon in development of colon tumors? K-ras codon mutations in normal colonic mucosa of patients with colorectal neoplasms. Chirurg 71: 1230-1234, 2000 (In German).

34. Weil R, Ohana G, Haplern M, Estlein D, Anvi A and Wolloch Y: Small nonpolypoid colorectal carcinoma. World J Surg 26: 503-508, 2002. 